\title{
Unsupervised and Adaptive Segmentation of Multispectral 3D Magnetic Resonance Images of Human Brain: A Generic Approach
}

\author{
Chahin Pachai ${ }^{1,3}$, Yue Min Zhu ${ }^{1}$, Charles R.G. Guttmann ${ }^{3}$, Ron Kikinis ${ }^{3}$, \\ Ferenc A. Jolesz ${ }^{3}$, Gérard Gimenez ${ }^{1}$, Jean-Claude Froment ${ }^{2}$, Christian Confavreux ${ }^{2}$, \\ and Simon K. Warfield ${ }^{3}$ \\ ${ }^{1}$ CREATIS, CNRS Research Unit UMR 5515, INSA de Lyon, France \\ \{pachai, zhu, gimenez\}@creatis.insa-lyon.fr \\ ${ }^{2}$ Departments of Neurology and Radiology-MRI, Hôpital Neurologique et \\ Neurochirurgical, Pierre Wertheimer, Lyon, France \\ \{christian.confavreux, jean-claude.froment\}@chu-lyon.fr

\footnotetext{
${ }^{3}$ Surgical Planning Laboratory, Department of Radiology-MRI, Brigham and Women's Hospital, Harvard Medical School, Boston, MA, USA \{guttmann, kikinis, jolesz, warfield\}bwh.harvard.edu
}

\begin{abstract}
A generic algorithm is presented for the segmentation of threedimensional multispectral magnetic resonance images. The algorithm is unsupervised and adaptive, does not require initialization, classifies the data in any number of tissue classes and suggests an optimal number of classes. It uses a statistical model including Bayesian distributions for brain tissues intensities and Gibbs Random Fields (GRF)-based spatial contiguity constraints. The classification is unsupervised, that is to say the intensity-based signatures of brain tissues and the spatial hyperparameters of the underlying GRF are derived from the data. Adaptivity is achieved through the variation of the size of the neighborhoods used for the estimation of the intensity characteristics. This allows slow variations of signal intensity in space to account for MRI intensity nonuniformity. Segmentation results with proton density, T2 and T1-weighted data are provided. The algorithm can be used as an independent segmentation module within a brain MRI data processing pipeline.
\end{abstract}

\section{Introduction}

The aim of this paper is to describe a generic algorithm for unsupervised segmentation of brain MR images, from which one could derive practical solutions to a variety of applications such as quantification and visualization of white matter (WM), gray matter (GM), cerebrospinal fluid (CSF) and pathological structures such as multiple sclerosis (MS) lesions and tumors. The goal of the present work is to provide a unique framework for the processing of brain data, a context in which several parameters may change from one application to the other. These parameters are the number of MRI input channels, their spatial resolutions, the number of brain tissues to be segmented, and the size of the smallest features to be segmented. The 
proposed framework supports multichannel MRI input, adaptive and unsupervised 3D segmentation based on Gibbs-Markov random fields (GRF-MRF). It accounts for the piecewise contiguity of brain regions (WM, GM) and, in a certain amount, for intensity nonuniformity without being dependent upon any specific initialization.

Markov Random Field (MRF) models were used with brain MR images in an important number of works to add spatial smoothness into the process of image segmentation [1-9]. These works reported that adding an explicit, local and low-level tissue-contiguity model within the segmentation framework could consistently improve the quality of the segmentation, especially in regions with low signal to noise ratio or corrupted by a strong white (salt and pepper) noise. However, these works did not identically address all the important issues. These issues are parameter estimation, cluster validation, problem dimensionality (MRI input channels, 2D or 3D implementation), the use of mixture models for partial volume effect correction, and whether the model accounts for intensity nonuniformity. In this contribution, we particularly stress on the integration of an MRF model within a completely unsupervised segmentation scheme. We briefly describe the statistical model, the resulting segmentation algorithm, the implementation issues and give visual results.

\section{Segmentation Model}

As described by Derin and Elliott in [10] and the related works [11-14] we defined a discrete 3D random field over a finite lattice of voxels and introduced a neighborhood system on it. A second order neighborhood system was used. The clique definition was similar to the one described by Derin et al. We did not consider single site cliques because we did not have prior information regarding the percentage of voxels in each region type (the missing prior information on the distribution of voxels in each tissue class could be obtained from a probabilistic atlas of human brain). This class of Gibbs distributions is used to characterize the spatial clustering of voxels into regions. It introduces prior spatial information regarding the size, the shape and the orientation of the regions to be segmented. The spatial smoothness constraint accounts for the natural contiguity of voxels belonging to the same tissue type, say WM, GM or CSF. If a voxel is of a certain tissue type, the neighboring voxels should also have a high probability of being of the same tissue type.

The segmentation vector or the scene was considered as a discrete random variable. This variable can be associated with a Gibbs Random Field defined on the lattice and with respect to the neighborhood system under conditions defined in [10]. The digital MRI data or the observation can be considered as a realization from a random field. At each voxel, the observation is a multidimensional vector, each component of which represents an MRI channel. The segmentation process consists of finding the scene realization (or segmentation), which produced the observation. We used a maximum a posteriori (MAP) criterion to estimate the realization, which maximized the a posteriori distribution. Using Bayes' theorem, the posterior probability was written as the sum of two contributions: the data term and the spatial term. The data term is the model providing a measurement given a scene realization (or 
segmentation) and the spatial term is the prior model of the spatial interaction between adjacent voxels. Both terms have intuitive meanings: the Gibbs term gives a prize for smoothness and the data term matches the fitted class means to the intensity.

At each voxel site, the observation vector can be modeled as the sum of the mean intensity of tissue class at that voxel and an additive, tissue (class) dependent, space variant, zero mean, Gaussian white noise. The diagonal elements of the associated covariance matrix (positive-definite symmetric) are the intensity variances and the off-diagonal elements are the correlation coefficients of the different input images. At each voxel site, the probability of observing an intensity given the tissue class label can be modeled with a multidimensional Gaussian function. To determine the data model (or the measurement model), tissue class parameters (means and covariances matrices) representing an intensity-based signature of the current segmentation were computed. The optimal segmentation was obtained by maximizing the posterior probability. Instead of finding the global minimum of this function, which is computationally prohibitive, we used Besag's Iterated Conditional Mode (ICM) to separate the segmentation process into two steps [15-16].

First, segmentation by maximization of the posterior probability: given the current estimates of the spatial and data models, a new segmentation is obtained at each voxel site, by minimizing the conditional density of equation 1 . This new segmentation only depends on the intensity estimates and the values of the neighboring voxels.

$$
\begin{aligned}
& F_{s}\left(x_{s} \mid y_{s}, x_{s^{\prime}}, s^{\prime} \in \eta_{s}\right)= \\
& \sum_{c \in C} V_{c}\left(x_{s} \mid \eta_{s}\right)+\frac{1}{2}\left\{\ln \left(\operatorname{det}\left(\sum_{x_{s}}\right)\right)+\left(y_{s}-\mu_{x_{s}}\right)^{T} \sum_{x_{s}}{ }^{-1}\left(y_{s}-\mu_{x_{s}}\right)\right\}
\end{aligned}
$$

where $x_{s}$ is the segmentation, $y_{s}$ the observation (multispectral MRI) at voxel site $s$, $\eta_{s}$ the neighborhood system centered at $s, c$ a clique belonging to clique ensemble $C$, $V_{c}$ the potentiel associated with clique type $c,\left(\mu_{x_{s}}, \Sigma_{x_{s}}\right)$ class dependent and spatially varying intensity functions (means and covariance matrices, respectively)

Second, estimation of the statistical model: given the current segmentation, new estimates of the spatial term and the data term are to be calculated. The data model is estimated by computing at each voxel site and for all tissue classes, means and covariance matrices are estimated locally using a neighborhood [17].

To estimate the hyperparameters of the prior model, Besag's Maximum PseudoLikelihood (MPL), the product of local conditional probabilities, was used [15-16], [6], [11]. The estimate of the current spatial model was obtained by maximizing the MPL product over all voxels. For a given segmentation, the pseudo-likelihood only depends on the current segmentation and the neighborhood system. An adaptive simulated annealing (SA) algorithm was used to minimize this function and find the 
hyperparameters. SA is a stochastic relaxation algorithm suitable to search the global minimum of a non convex function with many local minima.

The ICM algorithm is a partial optimal solution to the optimization problem and converges to a local minimum of the energy function. Therefore, the initialization issue has a major importance in the overall success of the segmentation. In order to initialize the algorithm, a one-class model was adopted. For a given tissue class number, all the clusters were split, one at a time, and the split which maximized the posterior probability was kept as the best segmentation. To increment the number of tissue classes, we split each tissue class in two, one at a time, to obtain different segmentations [6]. For splitting a given tissue class in two, we first split a given tissue class independently of the remaining voxels and then, using the resulting classification, we classified the data using a K-Means algorithm. This class-dependent initialization of the K-Means algorithm was repeated for all the current classes, so that each one initiated a different segmentation. The splitting algorithm is based on an initial division of a given cluster in two, following the principal component of the corresponding (multichannel) intensity distribution. The initial perturbation initiates a 2-Means algorithm (K-Means with 2 classes). The resulting classification initializes a global K-Means algorithm within the ICM cycle.

In order to find the optimal number of regions that gives the best fit (cluster validation issues), information criteria such as minimum description length [18], Akaike information criterion [19] or MAP criteria [6], [11] can be used. We considered a MAP criterion: the configuration that leads to the best segmentation is also the one which maximizes the MAP value over all the possibilities. Hence, cluster validation can be carried out along with the class splitting process.

\section{Implementation Issues}

The signal intensity of a given brain structure may exhibit important variations due to normal and pathological intra-tissue variations or the MRI system. These variations are slowly varying in space. The statistical model should accordingly allow the tissue classes to have slowly varying intensity functions. For this purpose, local estimates of means and covariance matrices at all voxel sites were computed over a neighborhood around each voxel. The size of this neighborhood is an important parameter for a good appreciation of intensity variations within a tissue class. To account for slowly varying (low frequency) phenomena, large parts of brain should be used for computing the estimations while accurate segmentation at the interfaces of tissue classes requires local estimates of intensity functions [17].

Local measures of means and covariance matrices for all tissue classes make the segmentation algorithm adaptive. In order to reduce the computation time, estimates were only calculated on a grid of points and the remaining values were computed using interpolation. The initial size of the neighborhood should be approximately equivalent to the size of the head and be progressively reduced, until a minimum size 
is reached. Since MRI data sets may have different resolutions, the size of the neighborhood should take into account this anisotropy.

For a given tissue class, the robustness of the estimations of its intensity functions at a given voxel site increases as the number of voxels with the same label and available in the neighborhood around that voxel grows. Estimates for a given class are then supposed valid if there are more than a minimal number of voxels with the same label in the neighborhood.

The spatial prior model based on a homogeneous and isotropic GRF has a major importance in regularizing the interfaces between different tissue classes. Increasing the value of the hyperparameters favors the clustering of voxels and imposes a stronger smoothness constraint. The relative difference between the data term and the Gibbs term has an impact both at the voxel level, for the computation of a new segmentation within the ICM and at the global level, where the MAP values are used to split the existing classes. We used an adaptive simulated annealing algorithm (ASA) implemented by Lester Ingber [20] to minimize Besag's maximum pseudolikelihood function. Even if estimating the hyperparameters by SA is time consuming, our experiments showed that changes in the values of hyperparameters might influence the outcome of the segmentation. Setting the hyperparameters to constant values may cause oversmoothing and undersmooting.

\section{Result}

Figure 1 shows the result of the segmentation of a double channel, PD and T2weighted data set. WM, GM and CSF are segmented and we further processed the data set to remove features which did not belong to the intracranial cavity. Figure 2 shows 3D reconstructions of WM, GM and CSF from a segmented millimetric T1weighted data set. Figure 3 shows the result of the segmentation of a millimetric T1weighted data set (one axial slice among 150). Successive segmentations are obtained as intermediary results. They are shown for 2, 3, 4 and 5 tissue classes. We also provide the segmentation prior to the iterations of the ICM algorithm. The resulting segmented images confirm the necessity of introducing spatial smoothness constraints through Gibbs prior. We can also notice the improvement of the overall segmentation result as the number of tissue classes increases. The best visual result was obtained with 5 tissue classes. This number was also suggested by the MAP stop criterion (cluster validation).

\section{Discussion and Conclusion}

The class of Gibbs distributions, used as a spatial prior, was an effective way to account for the piecewise contiguity of brain tissues. Data resolution, the number of tissue classes and the number of iterations within the ICM algorithm influenced the hyperparameter values. Our experiments confirmed that using multispectral MRI was 
an efficient way for more robust tissue identification. The adaptivity of the segmentation algorithm through the variation of the neighborhood size in estimating intensity functions allows slow intra-tissue variations of class intensities. In a certain amount, this ability accounts for intensity nonuniformity and normal or pathological intra-tissue intensity variations. Since the segmentation algorithm does not need to be initialized, any set of registered (if necessary) MRI channels can be used as input. The MAP-based cluster validation criterion provides a good indication for the best choice of the number of tissue classes to be segmented. The regularization model consistently and automatically improves the quality of the underlying segmentation. Reproducible measures and high quality visualization can be carried out using different MRI input configurations.

The implementation of this approach was useful to investigate the advantages and the limits of a completely unsupervised segmentation to reach both accuracy and reproducibility. The segmentation algorithm can be improved by incorporating a global model accounting for intensity nonuniformity. The low-level Markovian regularization process may be insufficient to successfully reconstruct strongly biased data. Using additional spatial prior from a probabilistic brain atlas after an atlas-topatient elastic matching step can also lead to more robust classification by assigning the GRF single site clique potentiels to relevant values derived from the atlas.

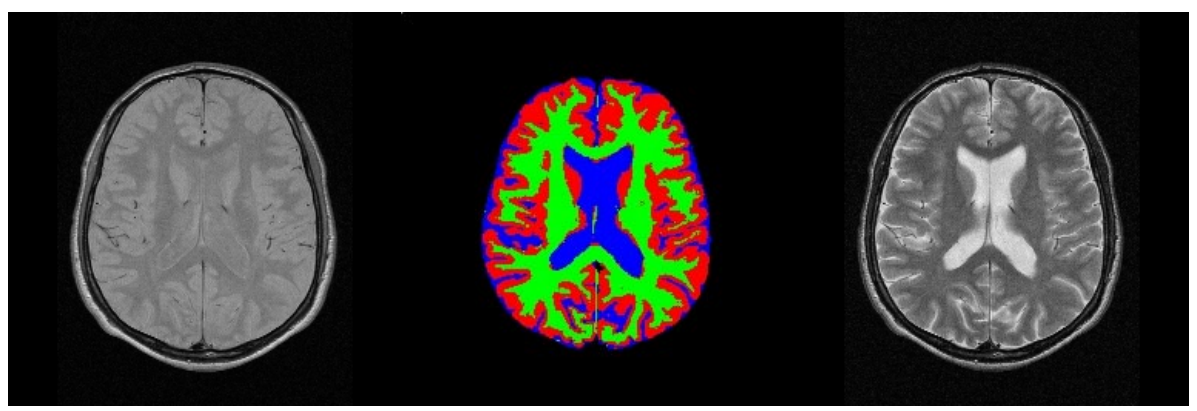

Fig. 1. Segmentation of double channel proton density (left) and T2-weighted (right) MRI. The intracranial cavity (WM, GM and CSF) is extracted from segmented data (middle).

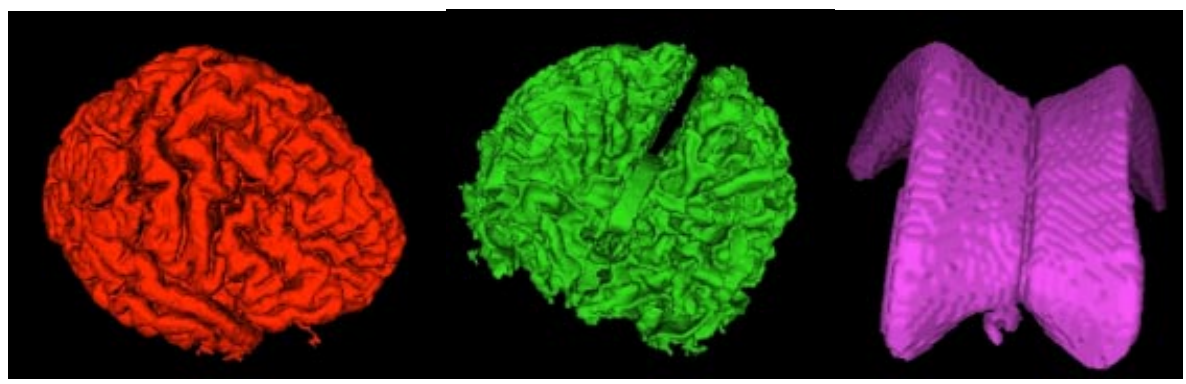

Fig. 2. 3D reconstruction of GM, WM and CSF from millimetric T1-weighted segmented data 

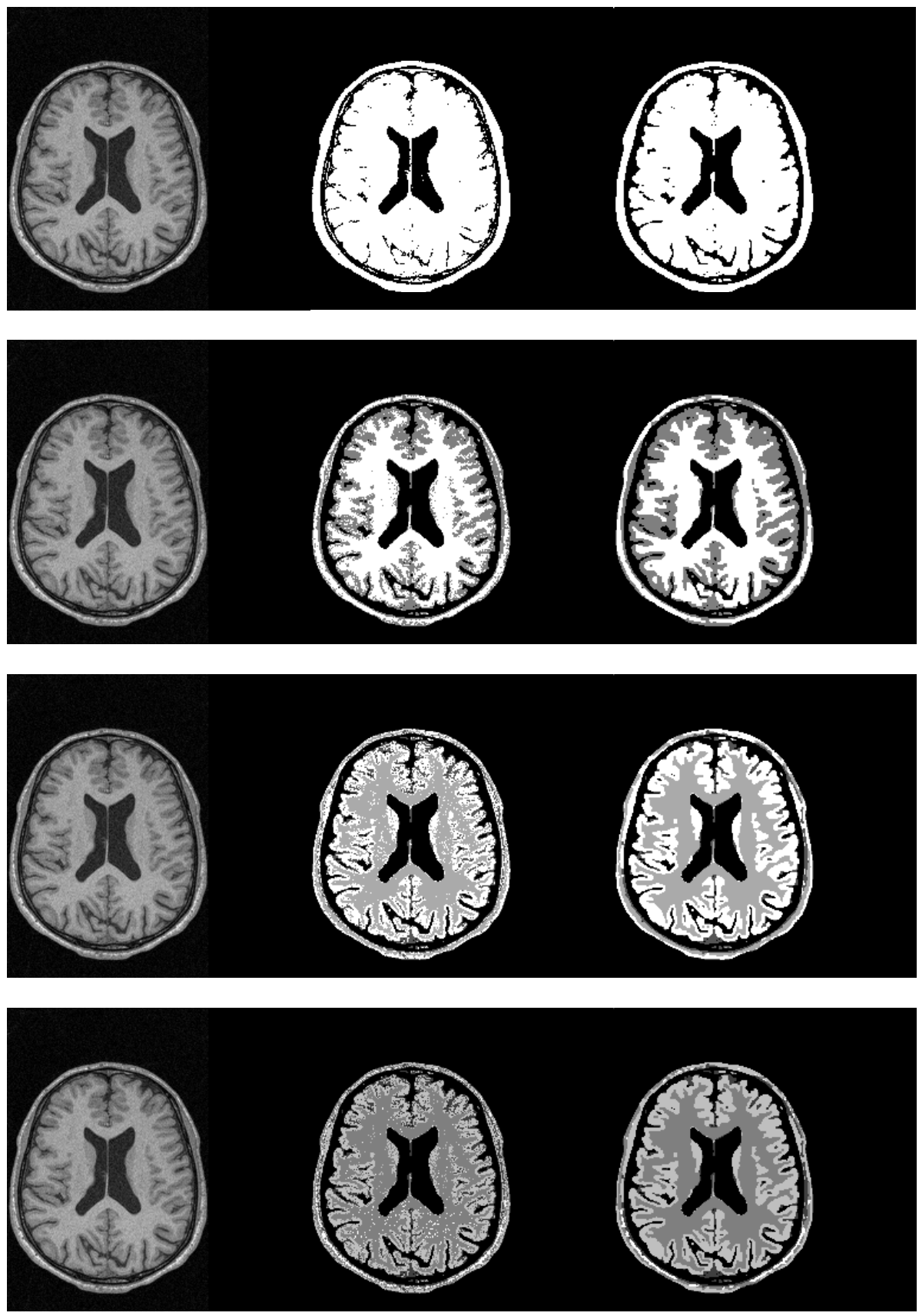

Fig. 3. Segmentation of single channel T1-weighted MRI in 2, 3, 4 and 5 tissue classes (topdown) : original image (left), segmentation without (middle column) and with (right column) MRF spatial regularization 


\section{References}

1. Hurn M.A., Mardia K.V., Hainsworth T.J., Berry E., Bayesian Fused Classification of Medical Images, IEEE Trans. Med. Imaging, 1996, vol 15, nº 6, p 850-858

2. Choi H.S., Haynor D.R., Kim Y., Partial Volume Tissue Classification of Multichannel Magnetic Resonance Images - A Mixel Model, IEEE Trans. Med. Imaging, 1991, vol 10, $\mathrm{n}^{\circ} 3, \mathrm{p}$ 395-407

3. Liang Z.R., MacFall J.R., Harrington D.P., Parameter estimation and tissue segmentation from multispectral MR images, IEEE Trans. Med. Imaging, 1994, vol. 13, no. 3, p 441-449

4. Ashton E.A., Berg M.J., Parker K.J., Weisberg J., Chen C.W., Ketonen L., Segmentation and feature extraction techniques, with applications to MRI head studies, Magn Reson Med, 1995, vol 33, n 5, p 670-677

5. Rajapakse J.C., DeCarli C., McLaughlin A., Giedd J.N., Krain A.L., Hamburger S.D., Rapoport J.L., Cerebral Magnetic Resonance Image Segmentation Using Data Fusion, J. Comput. Assist. Tomogr., 1996, vol 20, n², p 207-218

6. Fwu J.K., Djuric P.M., Unsupervised Vector Image Segmentation by a Tree Structure ICM Algorithm, IEEE Trans. Med. Imaging, 1996, vol 15, n 6, p 871-880

7. Chang M.M., Sezan M.I., Tekalp A.M., Berg M.J, Bayesian segmentation of multislice brain magnetic resonance imaging using three-dimensional Gibbsian priors, Optical Engineering, 1996, vol 35, $\mathrm{n}^{\circ} 11, \mathrm{p}$ 97-106

8. Held K., Rota Kops E., Krause B. J., Wells W. M., Kikinis R., Müller-Gärtner H.-W., Markov Random Field Segmentation of Brain MR Images, IEEE Trans. Med. Imaging, 1997, vol 16, n 6, p 878-886

9. Kapur T., Grimson W.E.L., Wells W.M., Kikinis R., Segmentation of Brain Tissue from Magnetic Resonance Images. Medical Image Analysis., 1996, vol 1, p 109-127

10. Derin H., Elliott H., Modeling and Segmentation of Noisy and Textured Images Using Gibbs Random Fields, IEEE Trans. on Pattern Analysis and Machine Intelligence, 1987, vol 9, $\mathrm{n}^{\circ} 1, \mathrm{p}$ 39-55

11. Won C.-S, Derin H., Unsupervised Segmentation of Noisy and Textured Images Using Markov Random Fields, Computer Vision Graphics and Image Processing, 1992, vol 54, $\mathrm{n}^{\circ} 4, \mathrm{p}$ 308-328

12. Derin H., Cole W.S., Segmentation of textured images using Gibbs random fields Computer Vision, Graphics, and Image Processing, 1986, vol 35, p 72-98

13. Derin H., Elliot H., Cristi R., Geman D., Bayes smoothing algorithms for segmentation of binary images modeled by markov random fields, IEEE Trans. on Pattern Analysis and Machine Intelligence, 1984, vol 6, $\mathrm{n}^{\circ}$ 6, $\mathrm{p}$ 707-720

14. Lakshmanan S., Derin H., Valid parameter space for 2-D Gaussian Markov random fields, IEEE Trans. Information Theory, 1993, vol 39, p 703-709

15. Besag J., Spatial interaction and the statistical analysis of lattice systems, J. R. Statist. Soc. B., 1974, vol 2, p 192-236

16. Besag J., On the statistial analysis of dirty pictures, J. R. Statist. Soc. B. , 1986, vol 48, $\mathrm{n}^{\circ}$ 3, p 259-302

17. Pappas T.N., An Adaptative Clustering Algorithm for Image Segmentation, IEEE Trans. Sig. Processing, 1992, vol 40, n 4, p 901-913

18. Liang Z., Jaszczak R.J., Coleman R.E., Parameter Estimation of Finite Mixtures Using the EM Algorithm and Information Criteria with Application to Medical Image Processing, IEEE Trans. Nuclear. Science, 1992, vol 39, n 4, p 1126-1133

19. Akaike H., A new look at the statistical model identification, IEEE Trans. Automat. Contr., 1974, vol. 19, p 716-723

20. Ingber L., Adaptive simulated annealing (ASA): Lessons learned, Control and Cybernetics, 1996, vol 25, p 33-54, URL : http://www.ingber.com/ 